\title{
Sexualidad juvenil: prácticas, actitudes y diferencias según sexo y variables de personalidad en universitarios chilenos
}

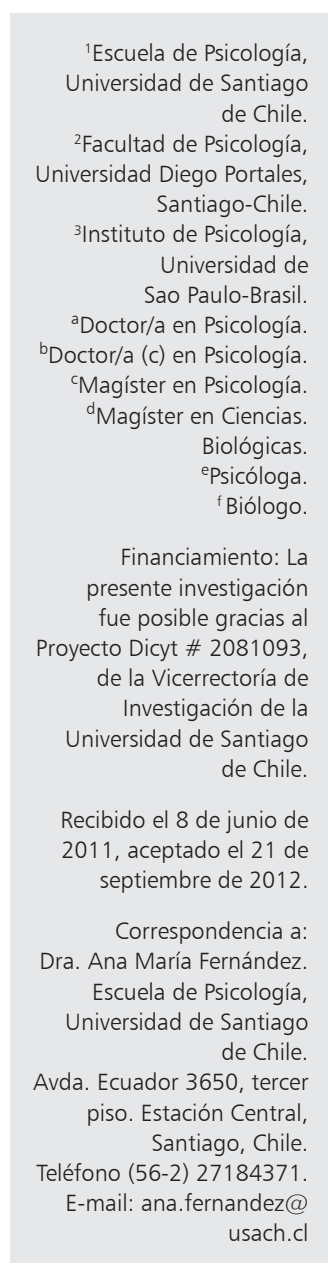

\author{
ANA MARÍA FERNÁNDEZ $Z^{1, \mathrm{a}, \mathrm{c}, \mathrm{e}}$, KAREM CELIS-ATENAS ${ }^{1, \mathrm{c}, \mathrm{e}}$, \\ NATALIA CÓRDOVA-RUBIO ${ }^{1, e}$, MICHELE DUFEY $^{2, b, d, e}$, \\ MARCO ANTÔNIO CORRÊA VARELLA 3 ,a,c,f, \\ JOSÉ HENRIQUE BENEDETTI PICCOLI FERREIRA ${ }^{3, b, c, f}$
}

\section{Youth sexuality: behaviors, attitudes and differences by sex and personality variables in Chilean university students}

Background: Sexual behavior usually begins in adolescence, and is mediated by biological, personality and socio-cultural variables which can affect the expression of preventive and risky sexual performance, as well as sex and age differences. Aim: To determine sex differences in the age of sexual initiation, the use of protective methods and mate selectivity in young men and women, as well as preventive practices according to age, and the prevalence of partner aggression. Subjects and Methods: Participants were 484 university students from public and private institutions, aged $22 \pm 3$ years (59\% women) assessed using a diversity of self-report measures of personality traits, romantic relationship quality, sexual role, attachment type, socio-sexual openness, and self-esteem; they also answered questions regarding sexual behavior, and violence. Results: Differences in age of sexual initiation, risky sexual behavior, and socio-sexual openness were observed between men and women. Aggression prevalence in romantic relationships also varied according to sex and age. Similarities and differences in patterns of behavior and personality variables were observed in relationship quality, sexual role, kindness, and responsibility in males and females. Conclusions: The findings present consistencies with the international evidence and differences that may be due to context specificities, providing also an empirical referent to consider in health planning.

(Rev Med Chile 2013; 141: 160-166).

Key words: Personality; Prevention \& Control; Sexuality.
E linicio de la adolescencia se asocia al desarrolo de las funciones sexuales y reproductivas en la dinámica física, biológica y psicológica de los individuos ${ }^{1,2}$ y la escasa experiencia expone a los jóvenes a contraer infecciones de transmisión sexual (ITS) y embarazo no deseado ${ }^{1,3}$. Factores individuales como la autoestima, personalidad, actitudes frente a las relaciones y los patrones de apego, influyen en la forma cómo se desarrolla la sexualidad. Por tanto, su estudio y análisis aporta en la comprensión y toma de decisiones desde las entidades de la salud ${ }^{4}$.

El conocimiento de la sexualidad se enriquece al trascender la descripción de la conducta juvenil $y$ se incorporan variables actitudinales y de personalidad relacionadas con el desarrollo sexual humano ${ }^{5,6}$.

La presente investigación se sustenta en reportes nacionales ${ }^{4,7}$ y la evidencia en torno a constructos psicológicos asociados a estrategias sexuales en 
la literatura internacional ${ }^{6,8,9,10}$. En primer lugar, se busca determinar: a) si el sexo masculino tendrá un inicio sexual más precoz que el femenino; b) si las mujeres utilizan preservativo en mayor medida que los hombres y si éstas son más selectivas que ellos en sus relaciones sexuales; c) si la cohorte más joven tendrá una mejor prevención que los individuos mayores y d) se indaga la prevalencia del ejercicio de violencia física y violencia verbal en esta población.

En segundo lugar, se exploran las diferencias sexuales en constructos psicológicos utilizados en la investigación intercultural (personalidad, calidad de la relación de pareja, rol sexual, tipo de apego, apertura sociosexual y autoestima) $)^{11,12}$, lo cual permitiría enriquecer la comprensión de la sexualidad juvenil en nuestro país. Los hallazgos resultarían de utilidad para la medicina y la salud pública, en la medida que la indagación psicológica colabora al entendimiento de diferencias sexuales, apoyada en evidencia para esta población de universitarios ${ }^{13}$.

\section{Material y Método}

\section{Muestra}

Se utilizó una muestra intencionada de universitarios en dos planteles públicos y cuatro privados de la zona central de Chile. Participaron 484 jóvenes $(41,3 \%$ hombres y $58,7 \%$ mujeres, $95 \%$ heterosexual) con una edad promedio de 22 años en hombres $(\mathrm{DE}=3,4)$ y 20,1 en mujeres $(\mathrm{DE}=2,6)$, y mediana de 20 y 21 años, respectivamente.

\section{Procedimiento}

Los estudiantes fueron invitados a participar voluntariamente del estudio por el profesor a cargo de la cátedra, a mediados del primer y segundo semestre académico del año 2007. Cada participante otorgó un consentimiento informado aprobado por la institución patrocinante. Luego se procedió a la administración de los instrumentos más preguntas específicas acerca de prácticas, experiencias, actitudes sexuales y violencia que han sido utilizadas en la investigación internacional ${ }^{14}$. La aplicación se realizó en una sala de clases, solicitando que se completaran de forma anónima, individual y sin conversar. Cada participante depositó sus datos en un sobre/caja que fue cerrado al finalizar para evitar aprehensiones respecto del anonimato.

\section{Instrumentos}

El Inventario de los Cinco Factores (BFI, 15, adaptado a Chile por 14) consta de 44 ítems que evalúan el modelo de los cinco factores de personalidad. Es necesario invertir los reactivos negativos (señalados con la letra R), para luego sumar todos los ítems por dimensión (se pueden dividir por el número de ítems si se desea estandarizarlos). Extraversión, ítems 1, 6R, 11, 16, 21R, 26, 31R y 36; Amabilidad, ítems 2R, 7, 12R, 17, 22, 27R, 32, 37R, 42; Responsabilidad, ítems 3, 8R, 13, 18R, 23R, 28, 33, 38, 43R; Neuroticismo, ítems 4, 9R, 14, 19, 24R, 29, 34R, 39; Apertura a la Experiencia, ítems 5, 10, 15, 20, 25, 30, 35R, 40, 41R, 44.

El Inventario de Componentes de las Relaciones (ICR, 16, adaptado a Chile por 17), contiene ítems con alta validez de facie en satisfacción, compromiso, intimidad, confianza, pasión y amor dentro de la relación. Se puntúan en una escala Likert de 1 (nada en absoluto) a 7 (completamente), cuyo total es la sumatoria del individuo para cada uno de los reactivos.

El Inventario de Roles Sexuales (IRS, 18, adaptado a Chile por 17) estima actitudes feministas, sumando los valores de cada reactivo e invirtiendo los ítems 1, 3, 5 y 7 . Se puntúa de 1 (completamente de acuerdo) a 7 (completamente en desacuerdo), sumando la puntuación bruta de los ítems, con puntajes altos indicando una mayor adherencia al feminismo y un rol no tradicional de la mujer.

El tipo de apego y su orientación al self y los otros (19, adaptado a Chile por 17) se evalúa mediante cuatro viñetas que describen los patrones prototípicos del vínculo romántico, en una escala Likert de "No me describe a mí con exactitud"1 a "Me describe a mí con mucha exactitud". El primero refiere al apego seguro, los siguientes ${ }^{2,3,4}$ apuntan al apego romántico inseguro, el 2 evalúa pérdida, el 3 preocupación y el cuarto apego temeroso. Para calcular el modelo del self se suman las puntuaciones de las preguntas 1 y 2 , restándole la suma de los ítems 3 y 4 . Para calcular el modelo de los otros, se suman los ítems $1+3$ y se le resta la suma del $2+4$.

El Inventario de Orientación Sociosexual (SOI), contiene siete reactivos que cuantifican el nivel de apertura a relaciones sexuales o una actitud positiva hacia el "sexo casual" y sin compromiso amoroso (20, adaptado a Chile por 17). Cada componente tiene una ponderación distinta (el ítem 7 se invierte) según la siguiente fórmula: 
$(5 \mathrm{X}$ ítem 1$)+(1 \mathrm{X}$ ítem 2$)+(5 \mathrm{X}$ ítem 3$)+(4 \mathrm{X}$ ítem 4$)+(2 \mathrm{X}$ el promedio de los ítems 5, 6 y 7$)$.

La Escala de Autoestima de Rosenberg (EAR) consta de 10 ítems (la mitad de éstos invertidos) que sirven como un indicador del bienestar psicológico general del individuo (adaptado a Chile por 21).

Todos los instrumentos alcanzaron adecuados niveles de consistencia interna siendo la confiabilidad de IRS relativamente baja, ameritando cautela en su interpretación (Tabla 1).

\section{Análisis de datos}

Para estimar las relaciones entre variables se calcularon pruebas de $\chi^{2}$, el coeficiente de correlación producto-momento $r$ de Pearson, pruebas $t$ de Student y ANOVAs. Para estimar la confiabilidad de las escalas, se utilizó el coeficiente alpha de Cronbach. Todos los análisis se ejecutaron con el software SPSS 18.0.

\section{Resultados}

Como se muestra en la Tabla 2, existen diferencias por sexo en las prácticas de los jóvenes. Las mujeres declararon salir con una sola persona en mayor medida que los hombres; ellos reportaron en mayor medida salir con varias personas o no tener compromiso y la experiencia de haber tenido relaciones sexuales fue superior en hombres que en mujeres. Por otra parte, las mujeres y los hombres han tenido en promedio 0,62 y 0,57 relaciones, respectivamente en menores de 20 años; y 1,25 y 2,18 , respectivamente, en los mayores. Se confirmó que las mujeres utilizan con mayor frecuencia protección en sus relaciones sexuales que los hombres a nivel general.

Por otro lado, los jóvenes mayores de 20 años superaron a los menores $(64,1 \%$ y $47,3 \%$ respectivamente) en el porcentaje de prácticas sexuales $\sin$ protección $\left(\chi^{2}=51,1, \mathrm{p}=, 000\right)$. Existen diferencias significativas en el porcentaje de relaciones sexuales sin protección entre las mujeres menores a 20 años $(37 \%)$ y mayores a esta edad $(60,8 \%$, $\left.\chi^{2}=14,8, \mathrm{p}=, 000\right)$, en tanto que en los hombres no se observó diferencias significativas por edad $(57,5 \%$ y $67,3 \%$, para menores y mayores de 20 años respectivamente, $\left.\chi^{2}=1,3, p=, 246\right)$. En la Figura 1, se muestran diferencias sexuales en la disposición de los jóvenes a tener un encuentro sexual según el tiempo que se conozca a la persona. Los hombres superan a las mujeres para todos los contextos, excepto en la búsqueda de una pareja estable, que fue similar para ambos sexos.

Tabla 1. Consistencia y medias para las variables de personalidad y diferencias individuales según sexo

\begin{tabular}{|c|c|c|c|c|c|}
\hline Dimensión evaluada & Confiabilidad $\alpha$ & Hombre & Mujer & Prueba $\mathbf{t}$ & Valor $p$ \\
\hline \multicolumn{6}{|l|}{$\mathrm{BFI}$} \\
\hline Extraversión & ,74 & $25,4 \quad(4,7)$ & $25,8 \quad(5,2)$ &,- 673 & ns \\
\hline Amabilidad &, 68 & $30,6 \quad(5,3)$ & $32,1 \quad(5,3)$ & $-3,071$ & 0,001 \\
\hline Responsabilidad & ,78 & $31,2 \quad(5,2)$ & $33,5 \quad(5,8)$ & $-4,541$ & 0,000 \\
\hline Neuroticismo &, 75 & $23,0 \quad(5,0)$ & $25,6 \quad(5,2)$ & $-5,507$ & 0,000 \\
\hline Apertura Experiencia & 81 & $40,0 \quad(6,4)$ & $39,1 \quad(6,3)$ & 1,506 & ns \\
\hline ICR &, 90 & $35,0 \quad(7,2)$ & $37,2 \quad(5,2)$ & $-2,980$ & 0,002 \\
\hline IRS &, 60 & $38,7 \quad(7,4)$ & $43,4 \quad(6,2)$ & $-7,423$ & 0,000 \\
\hline \multicolumn{6}{|l|}{ Apego } \\
\hline Seguro & $\mathrm{n} / \mathrm{a}$ & $4,4 \quad(1,6)$ & $4,5 \quad(1,6)$ &,- 702 & ns \\
\hline Self & - & $1,5 \quad(3,7)$ & $1,1 \quad(3,6)$ & 1,253 & ns \\
\hline Others & - & $0,4 \quad(3,6)$ & $0,8 \quad(3,5)$ & $-1,360$ & ns \\
\hline SOI & 80 & $41,5(20,1)$ & $21,8(12,7)$ & 11,513 & 0,000 \\
\hline EAR & 82 & $34,2 \quad(7,4)$ & $33,5 \quad(4,6)$ & 1,697 & $0,045^{*}$ \\
\hline
\end{tabular}

BFI: Cuestionario de Personalidad de los Cinco Grandes, ICR: Componentes de las Relaciones, IRS: Ideología de Roles Sexuales, SOI: Orientación Sociosexual, EAR: Escala de Autoestima de Rosenberg. *El valor t es significativo direccionalmente (con una sola cola de la distribución). 
Sexualidad juvenil - A. M. Fernández et al

Al ser consultados sobre el ejercicio de cualquier tipo de violencia en sus relaciones diádicas, un ANOVA significativo $\left(\mathrm{F}_{3,358}=3,891, \mathrm{p}=, 009\right)$ mostró efectos principales del sexo de los jóvenes $\left(\mathrm{F}_{1,358}=7,627, \mathrm{p}=, 006\right)$, con las mujeres reportando en promedio ser más violentas que los hombres $(\mathrm{M}$ $=4,6$ y 2,6, respectivamente). Efectos significativos de la edad $\left(\mathrm{F}_{1358}=4,115, \mathrm{p}=, 043\right)$ mostraron que los mayores de 20 años (hombres y mujeres) declaran frecuencias más altas de violencia que los menores ( $M=4,2$ y 2,8 , respectivamente), ver Figura 2 para diferencias por sexo. Específicamente, 26,8\% de los jóvenes señaló haber incurrido al menos una vez en algún tipo de violencia física, siendo 33,1\% quienes señalaron haber incurrido en al menos un evento de violencia verbal hacia su pareja (amenazas, insultos, gritos y chillidos). Además se observó una correlación baja, inversa y significativa entre autoestima y violencia verbal (cercana a un valor $\mathrm{r}$ de $-, 13, \mathrm{p}=, 012$ ) para ambos sexos.

Las diferencias sexuales en los constructos psicológicos estuvieron en concordancia con la literatura (Tabla 1). Las dimensiones de Amabilidad, Responsabilidad y Neuroticismo del BFI resultaron más altas en mujeres que en hombres, al igual que la Calidad de la Relaciones de Pareja (ICR) y la Ideología de Roles Sexuales Feministas (IRS). En autoestima (EAR) y apertura a la Sexualidad (SOI) los hombres obtuvieron puntuaciones más altas que las mujeres.

Adicionalmente se exploraron las diferencias intrasexuales en los constructos psicológicos en función del comportamiento sexual. Como muestran las Tablas 3 y 4, en ambos sexos para

Tabla 2. Diferencias por sexo en el inicio y la prevalencia de conductas sexuales en Universitarios

\begin{tabular}{|lccc|}
\hline Tipo de conducta & Hombre & Mujer & $\boldsymbol{\chi}^{\mathbf{2}}$ \\
\hline Saliendo con varias personas & $10,6 \%$ & $3,2 \%$ & $10,9^{* *}$ \\
\hline Saliendo con una persona (exclusivamente) & $31,8 \%$ & $48,1 \%$ & $12,7^{* *}$ \\
\hline Sin compromiso & $43,9 \%$ & $35,3 \%$ & $3,6^{*}$ \\
\hline Nunca ha tenido relaciones sexuales & $11,6 \%$ & $18,0 \%$ & $183^{* *}$ \\
\hline Ha tenido relaciones sexuales & $83,2 \%$ & $68,5 \%$ & $111,9^{* *}$ \\
\hline Ha tenido relaciones sin protección (sin preservativo) & $65,6 \%$ & $48,4 \%$ & $208^{* *}$ \\
\hline $\mathrm{n}$ & 198 & 283 & \\
\hline
\end{tabular}

Los valores de $\chi^{2}$ son significativos ${ }^{*} p<0,05$, a excepción de ${ }^{*} p=0,056$, que resulta marginal para dos colas, pero significativo para la hipótesis direccional.

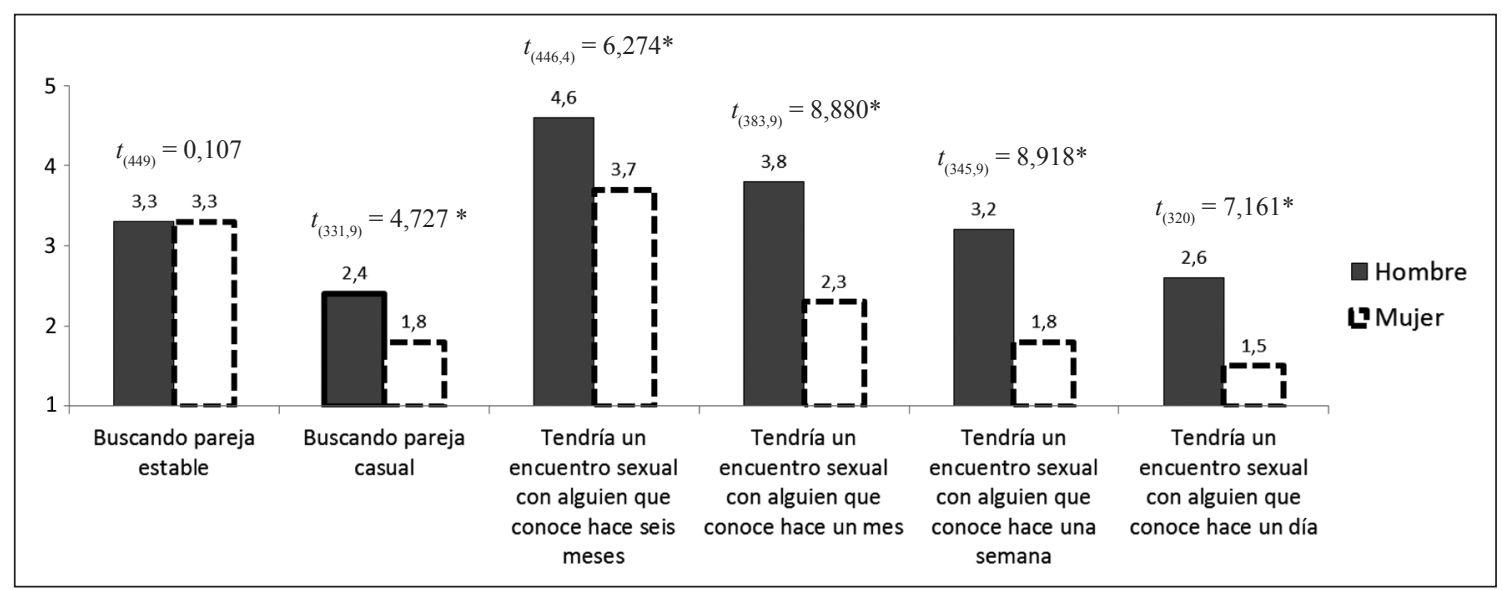

Figura 1. Promedio de adherencia a diferentes escenarios de búsqueda de pareja en hombres y mujeres. ${ }^{*} p<0,000$ con varianzas desiguales, prueba de LeveneFs $>$ 9,826. 


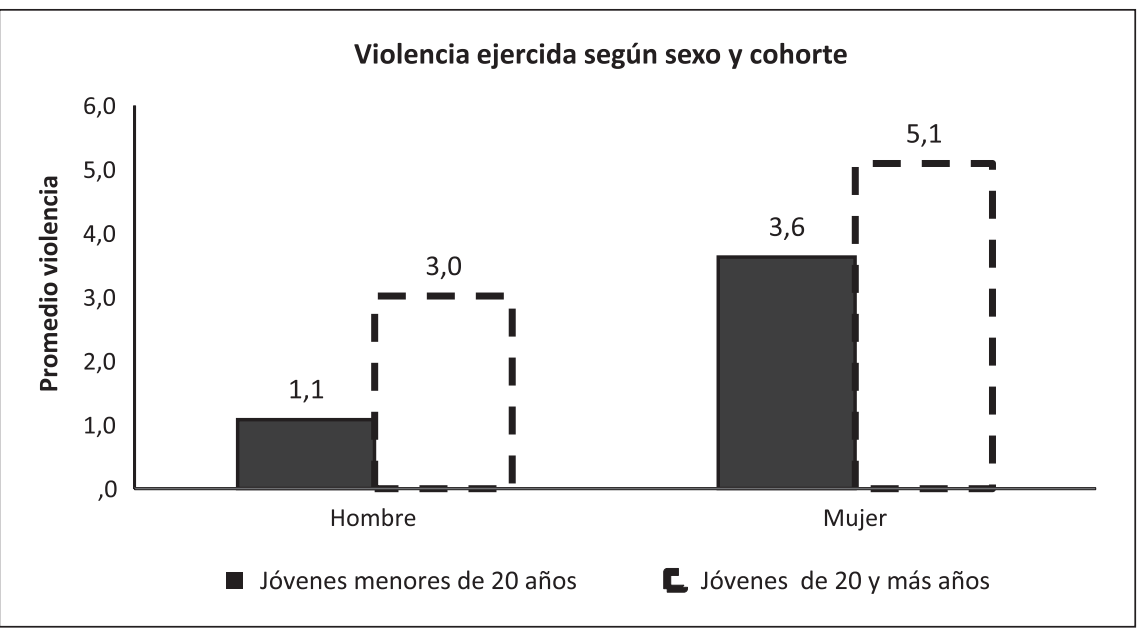

Figura 2. Diferencias en el promedio de violencia ejercida por sexo y grupo etario.

Tabla 3. Diferencias masculinas en los constructos psicológicos según la experiencia sexual y el uso de protección en las relaciones sexuales

\begin{tabular}{|c|c|c|c|c|c|c|}
\hline & \multicolumn{2}{|c|}{$\begin{array}{c}\text { Ha tenido relaciones } \\
\text { sexuales }\end{array}$} & \multicolumn{4}{|c|}{$\begin{array}{c}\text { Ha tenido sexo desprotegido } \\
\text { (sin preservativo) }\end{array}$} \\
\hline & Sí & No & $t$ & Sí & No & $t$ \\
\hline SOI & 44,1 & 18,0 & $\begin{array}{c}-8,668 \\
(p=, 000)\end{array}$ & 47,5 & 28,1 & $\begin{array}{c}6,685 \\
(p=, 000)\end{array}$ \\
\hline ICR & 35,5 & 21,7 & $\begin{array}{c}-3,488 \\
(p=, 001)\end{array}$ & & & \\
\hline IRS & 39,2 & 35,5 & $\begin{array}{c}-3,580 \\
(p=, 001)\end{array}$ & & & \\
\hline Respons. & & & & 30,3 & 32,9 & $\begin{array}{c}-3,392 \\
(p=, 001)\end{array}$ \\
\hline
\end{tabular}

Tabla 4. Diferencias femeninas en los constructos psicológicos según la experiencia sexual y el uso de protección en las relaciones sexuales

\begin{tabular}{|c|c|c|c|c|c|c|}
\hline & \multicolumn{2}{|c|}{$\begin{array}{c}\text { Ha tenido relaciones } \\
\text { sexuales }\end{array}$} & \multicolumn{4}{|c|}{$\begin{array}{l}\text { Ha tenido sexo desprotegido } \\
\text { (sin preservativo) }\end{array}$} \\
\hline & Sí & No & $t$ & Sí & No & $t$ \\
\hline SOI & 23,3 & 14,1 & $\begin{array}{c}-4,339 \\
(p=, 000)\end{array}$ & 25,2 & 18,4 & $\begin{array}{c}4,275 \\
(p=, 000)\end{array}$ \\
\hline ICR & 37,6 & 32,0 & $\begin{array}{c}-3,267 \\
(p=, 001)\end{array}$ & & & \\
\hline Amab. & 31,7 & 34,0 & $\begin{array}{c}2,801 \\
(p=, 005)\end{array}$ & 31,2 & 33,1 & $\begin{array}{l}-2,821 \\
(p=, 005)\end{array}$ \\
\hline
\end{tabular}

quienes se han iniciado en la vida sexual se presentan mayores niveles de SOI, a la vez que una mayor calidad de las relaciones de pareja (ICR). Asimismo, tanto en hombres como en mujeres se observó mayores niveles de SOI en quienes han tenido sexo desprotegido. Por otra parte, en el grupo de varones IRS fue mayor en quienes han tenido relaciones sexuales versus quienes no y la Responsabilidad fue mayor para quienes no han tenido sexo desprotegido. Por su parte, en las 
mujeres que no han tenido relaciones sexuales ni sexo desprotegido, se observó mayores niveles de Amabilidad.

\section{Discusión}

En función de los objetivos propuestos para este estudio, en primer lugar los hallazgos muestran que el inicio sexual de los jóvenes es más alto en hombres que en mujeres y que el sexo femenino utiliza métodos de protección en mayor medida que el masculino. Por otro lado, las mujeres tienen bajas intenciones de intimar sexualmente con alguien que conocen hace seis meses o un tiempo menor, siendo los hombres quienes declaran en mayor medida estar buscando un encuentro sexual casual (esto, pese a que en ambos sexos la búsqueda de una pareja estable es similar). En tercer lugar, se constata una mayor selectividad sexual femenina y un énfasis en maximizar las oportunidades sexuales por parte del sexo masculino, de forma consistente con los hallazgos de entidades nacionales ${ }^{7,22}$.

En cuanto al cuarto objetivo del estudio, se encontró que en las cohortes más jóvenes la prevención es superior a los de mayor edad y la protección en las relaciones sexuales femeninas supera al $60 \%$ en la muestra. Esto resulta coincidente con la predicción de mayor cuidado en la etapa de máximo potencial reproductivo y atractivo de las mujeres $^{5}$. Cabría preguntarse si estas diferencias etáreas se deben a que la generación más joven tiene una mejor educación sexual preventiva, o bien si al pasar los 20 años hay un cambio actitudinal y comportamental en torno a dichas prácticas. El esclarecer la causa de estas diferencias puede colaborar a la planificación sanitaria mediante la elaboración de estrategias de intervención preventivas específicas según un caso u otro. Finalmente, las mujeres reconocen mayor ejercicio de insultos, gritos y chillidos, e incluso agresiones físicas, pese a que en la literatura es el hombre quien ejecuta acciones violentas con resultados fatales o secuelas a largo plazo ${ }^{23}$. Según los resultados expuestos, la autoestima podría jugar un rol protector ante el ejercicio de la violencia verbal, lo cual se sugiere indagar en mayor profundidad en futuras investigaciones, por la contribución que ello podría tener en la planificación sanitaria.

Un factor común en los jóvenes, es que tanto la apertura a las relaciones sexuales sin compro- miso así como la percepción de que las relaciones de pareja son de mayor calidad, se da en mayor medida en hombres y mujeres que se han iniciado sexualmente. Esto tiene sentido si se considera la observación que la sexualidad juvenil en Chile se caracteriza por la experimentación y un aprendizaje orientado a la iniciación sexual ${ }^{24}$. Sería relevante conjugar este hallazgo con la promoción de un adecuado ejercicio de las prácticas preventivas sanitarias, a fines de potenciar el desarrollo psicológico integral y orientado al autocuidado en esta población.

Finalmente, y buscando dilucidar aquellos aspectos en que hombres y mujeres son distintos, se puede observar lo siguiente. En el caso de los hombres, es la variable Responsabilidad, es decir, una mayor autodisciplina y control de impulsos, la que permite diferenciar entre quienes usan o no protección (preservativo) en sus relaciones sexuales. En tanto que en las mujeres es la Amabilidad, esto es, su orientación prosocial y preocupación por la armonía social, la que permite distinguir quienes usan en mayor medida protección (preservativo), así como quienes no se han iniciado sexualmente. Este hallazgo reviste interés, debido a) al comportamiento sexualmente diferenciado que tienen ambas variables de personalidad, lo cual implicaría abordajes diferenciados en la intervención sanitaria; $b$ ) al posible rol protector que Responsabilidad y Amabilidad tendrían en el autocuidado de hombres y mujeres respectivamente; $y$ c) el introducir a la investigación de la sexualidad variables de personalidad que tradicionalmente se han estudiado fuera de ella.

En conclusión, a través de este estudio se ha podido describir el comportamiento sexual juvenil en aspectos que se diferencian por sexo y edad. Asimismo, se logra identificar variables psicológicas vinculadas a las relaciones interpersonales y la personalidad, que contribuyen a un mayor entendimiento de la dinámica psicológica juvenil y el autocuidado o el nivel de riesgo en torno al inicio y ejercicio de la vida sexual. Ello contiene un potencial importante a ser considerado en el diseño de estrategias sanitarias preventivas y promocionales específicas.

\section{Referencias}

1. Centro Latinoamericano y Caribeño de Demografía (CELADE). Juventud, población y desarrollo en América 
Latina y el Caribe. Comité Especial sobre Población y Desarrollo del Período de Sesiones de la CEPAL sesionó los días 3 y 4 de abril de 2000.

2. Hein K, Cárdenas A. Perspectivas de Juventud en el imaginario de la Política Pública. Revista Última Década 2009; 30: 95-120.

3. Aravena A, Fritz H. Sexualidad juvenil y exposición a riesgos sanitarios. Una aproximación desde los imaginarios sociales y el enfoque de género. Revista Observatorio de la Juventud 2010; 27: 23-37.

4. Dides C, Morán JM, Benavente MC, Pérez MS. Salud sexual y reproductiva en Chile 2007: actualización de datos estadísticos/ FLACSO-Chile. Programa de Género y Equidad. Santiago, Chile: FLACSO 2008.

5. Buss DM. Sex differences in human mate preferences: Evolutionary Hypotheses tested in 37 cultures. Behav Brain Sci 1989; 12: 1-49.

6. Schmitt DP, Buss DM. Sexual dimensions of person description: beyond or subsumed by the Big Five? J Res Pers 2000; 34: 141-77.

7. INJUV, Instituto Nacional de la Juventud. VI Encuesta Nacional de Juventud. Santiago, Chile 2010.

8. Buss DM. The Great Struggles of Life: Darwin and the Emergence of Evolutionary Psychology. Am Psychol 2009; 64: 140-8.

9. Schmitt DP, Realo A, Voracek M, Allik J. Why can't a man be more like a woman? Sex differences in big five personality traits across 55 cultures. J Pers Soc Psychol 2008; 94: 168-82.

10. Schmitt DP, Shackelford TK. Big Five traits related to short-term mating: From personality to promiscuity across 46 nations. Evol Psychol 2008; 6: 246-82.

11. Schmitt DP. Sociosexuality from Argentina to Zimbabwe: a 48-nation study of sex, culture, and strategies of human mating. Behav Brain Sci 2005; 28: 247-311.

12. Jara G, Molina T, Caba F, Molina R. Sexualidad en alumnos universitarios. Centro de Medicina Reproductiva y Desarrollo Integral del Adolescente (CEMERA), Facultad de Medicina Universidad de Chile Santiago 1997.
13. Buss DM, Hawley P. The evolution of personality and individual differences. New York, NY: Oxford University Press; 2011.

14. Schmitt DP and 118 members of the Personality Description Project. Are men universally more dismissing than women? Gender differences in romantic attachment across 62 cultural regions. Pers Relatsh 2003; 10: 309-33.

15. McCrae R, Costa P. The NEO Personality Inventory: Using the Five-Factor Model in Counseling. J Couns Dev 1991; 69: 367-72.

16. Fletcher GJ, Simpson JA, Thomas G. The measurement of perceived relationship quality components: A confirmatory factor analytic approach. Pers Soc Psychol Bull 2000; 26: 340-54

17. Schmitt DP and 121 members of the International Sexuality Description Project. Patterns and universals of mate poaching across 53 nations: The effects of sex, culture, and personality on romantically attracting another person's partner. J Pers Soc Psychol; 86: 560-84.

18. Burkhe RA. Factor dimensions across different measures of sex role ideology. Sex Roles 1988; 18: 309-21.

19. Bartholomew K, Horowitz L. Attachment styles among young adults: a test of four category model. J Pers Soc Psychol 1991; 61: 226-44.

20. Simpson J, Gangestad SW. Individual differences in sociosexuality: Evidence for convergent and discriminant validity. J Pers Soc Psychol 1991; 60: 870-83.

21. Rojas-Barahona CA, Zegers B, Förster CE. La escala de Autoestima de Rosenberg: validación para Chile en una muestra de jóvenes adultos, adultos y adultos mayores. Rev Med Chile 2009; 137: 791-800.

22. INJUV, Instituto Nacional de la Juventud. V Encuesta Nacional de Juventud. Santiago, Chile 2007.

23. Miner E, Shackelford T, Starrat V. Mate value of romantic partners predicts men's partner-directed verbal insults. Pers Individ Dif 2009; 46: 135-9.

24. Barrientos J. ¿Nuevas normatividad del comportamiento sexual juvenil en Chile? Última Década 2006; 24: 85-101. 